International Mathematical Forum, 1, 2006, no. 12, 563-576

\title{
MULTIENDOMORPHISMS OF HYPERGROUPOIDS
}

\author{
Giuseppe Gentile \\ Department of Mathematics \\ University of Messina \\ Contrada Papardo - Salita Sperone, 31 \\ 98166 Messina - ITALY
}

\begin{abstract}
We introduce the notion of multiendomorphism in a hypergroupoid and the notion of G-semiring. We show that, if $(H, *)$ is a commutative semi-hypergroup, these multiendomorphisms form a G-semiring $(E,+, \circ, \leq)$, where the operation + is induced by $*, \circ$ is the usual composition of maps $\circ$ and $\leq$ is the usual inclusion of maps. Moreover, we show under which conditions the G-semiring $(E,+, \circ, \leq)$ is, in fact, a semiring.
\end{abstract}

Mathematics Subject Classification: Primary 20N20; Secondary 05F06, $16 \mathrm{Y} 60$.

Keywords: Multiendomorphism, semiring, G-semiring.

\section{Definitions and recalls}

First of all we introduce some definitions of hypergroup theory (see [1] for more details).

Definition 1.1 A hypergroupoid $(H, *)$ is a non-empty set $H$ equipped with a hyperoperation $*$, that is a map $*: H \times H \rightarrow \mathcal{P}^{*}(H)$, where $\mathcal{P}^{*}(H)$ is the set of non-empty subsets of $H$. If $x, y \in H$, we will denote by $x * y$ the hyperproduct of $x$ and $y$. For $A, B \subseteq H$ we define, $A \circ B=\bigcup_{a \in A, b \in B} a * b$.

Definition 1.2 A hypergroupoid $(H, *)$ is said to be semi-hypergroup if and only if:

$$
\forall\left(x_{1}, x_{2}, x_{3}\right) \in H^{3}, x_{1} *\left(x_{2} * x_{3}\right)=\left(x_{1} * x_{2}\right) * x_{3} .
$$


A hypergroupoid $(H, *)$ is said to be commutative if and only if:

$$
\forall x, y \in H, x * y=y * x .
$$

A hypergroupoid $(H, *)$ is said to be reproductive (or quasi-hypergroup) if and only if:

$$
\forall x \in H, x * H=H=H * x .
$$

A reproductive semi-hypergroup is said to be a hypergroup.

Example 1.1 Let's consider the hypergroupoid $(H, *)$ defined by the following table:

\begin{tabular}{c|c|c|c|}
$*$ & 1 & 2 & 3 \\
\hline 1 & 1 & 1,2 & $1,2,3$ \\
\hline 2 & 1 & 1,2 & $1,2,3$ \\
\hline 3 & 1 & 1,2 & 3 \\
\hline
\end{tabular}

$(H, *)$ is a semi-hypergroup, neither commutative nor reproductive.

Example 1.2 The hypergroupoid $(H, *)$ defined by the following table:

\begin{tabular}{c|c|c|}
$*$ & 1 & 2 \\
\hline 1 & 1 & 1,2 \\
\hline 2 & 2 & 1 \\
\hline
\end{tabular}

is a quasi-hypergroup, neither commutative nor associative.

Example 1.3 The hypergroupoid $(H, *)$ defined by the following table:

\begin{tabular}{c|c|c|c|}
$*$ & 1 & 2 & 3 \\
\hline 1 & 1 & 1,2 & 1,3 \\
\hline 2 & 1,2 & 2 & 2,3 \\
\hline 3 & 1,3 & 2,3 & 3 \\
\hline
\end{tabular}

is a commutative hypergroup.

Definition 1.3 Let $(H, *),\left(H^{\prime}, *^{\prime}\right)$ be two hypergroupoids; a map $f: H \rightarrow$ $H^{\prime}$ is said to be a homomorphism if and only if:

$$
\forall x, y \in H, \quad f(x * y) \subseteq f(x) *^{\prime} f(y) ;
$$

$f$ is said to be a good homomorphism if and only if:

$$
\forall x, y \in H, \quad f(x * y)=f(x) *^{\prime} f(y) .
$$

If $(H, *)=\left(H^{\prime}, *^{\prime}\right), f$ is said to be an endomorphism (resp. good endomorphism). Moreover, we will denote by $E(H, *)$, or simply $E(H)$, the set of all endomorphisms of $(H, *)$ and by $E_{g}(H, *)$, or simply $E_{g}(H)$, the set of all good endomorphisms of $(H, *)$. 
Now we generalize the previous notion of homomorphism and that one of multihomomorphism given in [2] by the following:

Definition 1.4 Let $(H, *),\left(H^{\prime}, *^{\prime}\right)$ be two hypergroupoids; a map $f: H \rightarrow$ $\mathcal{P}^{*}\left(H^{\prime}\right)$ is said to be a multihomomorphism if and only if:

$$
\forall x, y \in H, \quad f(x * y) \subseteq f(x) *^{\prime} f(y) ;
$$

$f$ is said to be a good multihomomorphism if and only if:

$$
\forall x, y \in H, \quad f(x * y)=f(x) *^{\prime} f(y) .
$$

If $(H, *)=\left(H^{\prime}, *^{\prime}\right)$, $f$ is said to be a multiendomorphism (resp. good multiendomorphism). Moreover, we will denote by $\mathcal{E}(H, *)$, or simply $\mathcal{E}(H)$, the set of all multiendomorphisms of $(H, *)$ and by $\mathcal{E}_{g}(H, *)$, or simply $\mathcal{E}_{g}(H)$, the set of all good multiendomorphisms of $(H, *)$.

Remark 1.1 If $(H, *)$ is hypergroupoid with $H=\left\{h_{1}, h_{2}, \ldots, h_{n}\right\}$, the multiendomomorphism $f \in \mathcal{E}(H)$ such that:

$$
\left\{\begin{array}{l}
f\left(h_{1}\right)=A_{1} ; \\
f\left(h_{2}\right)=A_{2} ; \\
\vdots \\
f\left(h_{n}\right)=A_{n}
\end{array}\right.
$$

with $A_{i} \subseteq H, \forall i=1, \ldots, n$, will be denoted by:

$$
\left(A_{1}, A_{2}, \ldots, A_{n}\right) \text {. }
$$

If $A_{i}=\{a\}$ we will write a instead of $\{a\}$.

Remark 1.2 Clearly any good homomorphism (resp. good multihomomorphism) is a homomorphism (resp. multihomomorphism); in particular, we obtain that in any hypergroupoid $(H, *)$ we have:

$$
E_{g}(H) \subseteq E(H), \quad \mathcal{E}_{g}(H) \subseteq \mathcal{E}(H)
$$

Moreover, by identifying the map $f: x \mapsto f(x)$ whit the map $f: x \mapsto\{f(x)\}$, it is clear that any homomorphism (resp. good homomorphism) is a multihomomorphism (resp. good multihomomorphism); so, the following inclusions are true:

$$
E_{g}(H) \subseteq \mathcal{E}_{g}(H), \quad E(H) \subseteq \mathcal{E}(H) .
$$

Then, in any hypergroupoid $(H, *)$ we have:

$$
E_{g}(H) \subseteq E(H) \subseteq \mathcal{E}(H), \quad E_{g}(H) \subseteq \mathcal{E}_{g}(H) \subseteq \mathcal{E}(H)
$$

Notice that, in general,

$$
E(H) \nsubseteq \mathcal{E}_{g}(H), \quad \mathcal{E}_{g}(H) \nsubseteq E(H)
$$


The preceding inclusions are not invertible as we can see in the following example.

Example 1.4 Let $(H, *)$ be the hypergroupoid defined by the following table:

\begin{tabular}{c|c|c|}
$*$ & 1 & 2 \\
\hline 1 & 1,2 & 1 \\
\hline 2 & 1 & 2 \\
\hline
\end{tabular}

We have:

$$
\begin{aligned}
E_{g}(H) & =\{(1,2),(2,2)\} \\
E(H) & =\{(1,1),(1,2),(2,2)\}=E_{g}(H) \cup\{(1,1)\} \\
\mathcal{E}_{g}(H) & =\{(1,2),(2,2),(H, 2),(H, H)\}=E_{g}(H) \cup\{(H, 2),(H, H)\} \\
\mathcal{E}(H) & =\{(1,1),(1,2),(1, H),(2,2),(H, 1),(H, 2),(H, H)\}= \\
& =\mathcal{E}_{g}(H) \cup\{(1,1),(1, H),(H, 1)\}= \\
& =E(H) \cup\{(1, H),(H, 1),(H, 2),(H, H)\}
\end{aligned}
$$

Moreover, $E(H)$ and $\mathcal{E}_{g}(H)$ are not comparable.

\section{The G-semirings}

In this section we recall the definition of semiring (see [5] for more details) and we give the notion of $G$-semiring.

Definition 2.1 An algebraic structure $(S,+, \times)$ is said to be a semiring if and only if the following conditions are satisfied:

1. $(S,+)$ is a semigroup;

2. $(S, \times)$ is a semigroup;

3. The distributivity law holds for any triple of elements of $S$.

If $(S,+)$ has an identity 0 and $(S, \times)$ has an identity 1 , with $0 \neq 1$, the semiring is said to be with identities.

Example 2.1 The most natural example of semiring is $(\mathbf{N},+, \times)$, where $\mathbf{N}$ is the set of positive integers, + and $\times$ are the natural addition and multiplication.

We recall the following definition (see [6]). 
Definition 2.2 An algebraic structure $(G, \cdot, \leq)$ is said to be a partially ordered groupoid if and only if:

1. $(G, \cdot)$ is a groupoid;

2. $(G, \leq)$ is a partially ordered set;

3. $a \leq b \Rightarrow a \cdot c \leq b \cdot c, c \cdot a \leq c \cdot b, \forall c \in G$.

If the operation - is associative $(G, \cdot, \leq)$ is said to be a partially ordered semigroup.

Now we can give the following notion.

Definition 2.3 An algebraic structure $(S,+, \times, \leq)$ is said to be a $G$-semiring if and only if the following conditions are satisfied:

$S 1 . \quad(S,+, \leq)$ is a partially ordered semigroup;

S2. $(S, \times, \leq)$ is a partially ordered semigroup;

S3. $\forall x, y, z \in S, \quad\left\{\begin{array}{l}x \times(y+z) \leq(x \times y)+(x \times z) \\ (x+y) \times z \leq(x \times z)+(y \times z)\end{array}\right.$.

If $(S,+)$ has an identity 0 and $(S, \times)$ has an identity 1 , with $0 \neq 1$, the $G$ semiring is said to be with identities.

Remark 2.1 Clearly any semiring (with identities) is a G-semiring (with identities), where the partial order is the equality. The converse is not true; an example will be given in the next section.

Remark 2.2 If in a $G$-semiring $(S,+, \times, \leq)$ the following conditions are satisfied:

$$
\forall x, y, z \in S, \quad\left\{\begin{array}{l}
(x \times y)+(x \times z) \leq x \times(y+z) \\
(x \times z)+(y \times z) \leq(x+y) \times z
\end{array},\right.
$$

then $(S,+, \times)$ is a semiring.

\section{The G-semiring of multiendomorphisms}

In this section we will construct a $G$-semiring involving multiendomorphisms of commutative semi-hypergroups, showing that, in general, this $G$-semiring is not a semiring.

Lemma 3.1 Let $(H, *)$ be a hypergroupoid, $A, B \subseteq H, f \in \mathcal{E}(H)$. Then $f(A * B) \subseteq f(A) * f(B)$. 
Proof.

$$
\begin{aligned}
f(A * B) & =f\left(\bigcup_{a \in A, b \in B} a * b\right)=\bigcup_{a \in A, b \in B} f(a * b) \subseteq \\
& \subseteq \bigcup_{a \in A, b \in B}(f(a) * f(b))=\left(\bigcup_{a \in A} f(a)\right) *\left(\bigcup_{b \in B} f(b)\right)= \\
& =f(A) * f(B) .
\end{aligned}
$$

$\diamond$

In a similar way one can prove the following lemma.

Lemma 3.2 Let $(H, *)$ be a hypergroupoid, $A, B \subseteq H, f \in \mathcal{E}_{g}(H)$. Then $f(A * B)=f(A) * f(B)$.

Now we can prove the following proposition.

Proposition 3.3 Let $(H, *)$ be a hypergroupoid, $f, g \in \mathcal{E}(H)$. Then $f \circ g \in$ $\mathcal{E}(H)$, where o is the usual composition of maps.

Proof. From $f, g \in \mathcal{E}(H)$ it follows that:

$$
\forall x, y \in H, \quad f(x * y) \subseteq f(x) * f(y), g(x * y) \subseteq g(x) * g(y)
$$

and then:

$$
f \circ g(x * y)=f(g(x * y)) \subseteq f(g(x) * g(y)) .
$$

By Lemma 3.1 it follows that:

$$
f(g(x) * g(y)) \subseteq f(g(x)) * f(g(y))=(f \circ g)(x) *(f \circ g)(y)
$$

that is $f \circ g \in \mathcal{E}(H)$. $\diamond$

By Lemma 3.2 and using a similar argument one can prove the following proposition.

Proposition 3.4 Let $(H, *)$ be a hypergroupoid, $f, g \in \mathcal{E}_{g}(H)$. Then $f \circ g \in$ $\mathcal{E}_{g}(H)$.

Now we can prove that.

Theorem $3.5(\mathcal{E}(H), \circ)$ and $\left(\mathcal{E}_{g}(H), \circ\right)$ are semigroups with identity. 
Proof. By the previous propositions $(\mathcal{E}(H), \circ)$ and $\left(\mathcal{E}_{g}(H), \circ\right)$ are groupoids. Clearly, the associative law holds for any triple of (good) multiendomorphisms. Moreover, if we consider the map $1_{H}: x \mapsto\{x\}$ we have:

$$
\forall x, y \in H, \quad 1_{H}(x * y)=x * y=1_{H}(x) * 1_{H}(y)
$$

that is $1_{H}$ is a good multiendomorphism (and so a multiendomorphism). Finally:

$\forall f \in \mathcal{E}(H), \forall x \in H,\left(f \circ 1_{H}\right)(x)=f\left(1_{H}(x)\right)=f(x)=1_{H}(f(x))=\left(1_{H} \circ f\right)(x)$,

and so $1_{H}$ is the identity in $(\mathcal{E}(H), \circ)$ (and therefore in $\left(\mathcal{E}_{g}(H), \circ\right)$ also). $\diamond$

Let $(H, *)$ be a hypergroupoid. We introduce in $\mathcal{E}(H)$ (and therefore in $\left.\mathcal{E}_{g}(H)\right)$ another operation + , called "sum", defined by setting:

$$
\forall f, g \in \mathcal{E}(H), \forall x \in H, \quad(f+g)(x)=f(x) * g(x)
$$

In general, the sum of multiendomorphisms is not a multiendomorphism as we can see in the following example.

Example 3.1 Let $(H, *)$ be the hypergroupoid defined by the following table:

\begin{tabular}{c|c|c|}
$*$ & 1 & 2 \\
\hline 1 & 2 & 1,2 \\
\hline 2 & 1,2 & 1 \\
\hline
\end{tabular}

We have:

$$
\mathcal{E}(H)=\{(1,1),(1,2),(2,1),(2,2),(H, H)\} .
$$

If we consider $f=(1,1)$ and $g=(1,2)$, we obtain that:

$$
\begin{aligned}
& (f+g)(1)=f(1) * g(1)=1 * 1=2 ; \\
& (f+g)(2)=f(2) * g(2)=1 * 2=H
\end{aligned}
$$

that is:

$$
f+g=(2, H)
$$

and so $f+g \notin \mathcal{E}(H)$.

In which case the sum of multiendomorphisms is a multiendomorphism? The answer is given in the following proposition.

Proposition 3.6 Let $(H, *)$ be a commutative semi-hypergroup, $f, g \in \mathcal{E}(H)$. Then $f+g \in \mathcal{E}(H)$. 
Proof. Let $x, y \in H, f, g \in \mathcal{E}(H)$. Then, by definition of + we have:

$$
(f+g)(x * y)=\bigcup_{z \in x * y}(f+g)(z)=\bigcup_{z \in x * y} f(z) * g(z)
$$

but, clearly:

$$
\bigcup_{z \in x * y} f(z) * g(z) \subseteq \bigcup_{t, z \in x * y} f(t) * g(z)=\left(\bigcup_{t \in x * y} f(t)\right) *\left(\bigcup_{z \in x * y} g(z)\right)
$$

the last hyperproduct is $f(x * y) * g(x * y)$; now, by hypotesis we have:

$$
f(x * y) * g(x * y) \subseteq(f(x) * f(y)) *(g(x) * g(y)) ;
$$

by hypotesis, it follows that:

$$
(f(x) * f(y)) *(g(x) * g(y))=(f(x) * g(x)) *(f(y) * g(y))
$$

the last hyperproduct is, by definition, $(f+g)(x) *(f+g)(y)$. By the previous equalities and inclusions we obtain:

$$
(f+g)(x * y) \subseteq(f+g)(x) *(f+g)(y),
$$

that is $f+g \in \mathcal{E}(H)$.

Remark 3.1 Unlike multiendomorphisms, in general the sum of good multiendomorphisms is not a good multiendomorphism also in the case that $(H, *)$ is a commutative semi-hypergroup, as we can see in the following example.

Example 3.2 Let $(H, *)$ be the commutative hypergroup defined by the following table:

\begin{tabular}{c|c|c|c|}
$*$ & 1 & 2 & 3 \\
\hline 1 & 1 & 2 & 3 \\
\hline 2 & 2 & 1 & 3 \\
\hline 3 & 3 & 3 & 1,2 \\
\hline
\end{tabular}

The map $1_{H}=(1,2,3)$ is clearly a good multiendomorphism; let $f=1_{H}+1_{H}$; we have:

$$
\begin{aligned}
& f(1)=\left(1_{H}+1_{H}\right)(1)=1_{H}(1) * 1_{H}(1)=1 * 1=1 ; \\
& f(2)=\left(1_{H}+1_{H}\right)(2)=1_{H}(2) * 1_{H}(2)=2 * 2=1 ; \\
& f(3)=\left(1_{H}+1_{H}\right)(3)=1_{H}(3) * 1_{H}(3)=3 * 3=\{1,2\} ;
\end{aligned}
$$

that is:

$$
f=(1,1,\{1,2\})
$$


This map is not a good multihomomorphism because, for instance

$$
f(3 * 3)=1,
$$

while:

$$
f(3) * f(3)=\{1,2\} .
$$

So, $1_{H} \in \mathcal{E}_{g}(H)$, while $1_{H}+1_{H} \notin \mathcal{E}_{g}(H)$. Direct calculations show that the set of all good multihomomorphisms is:

$$
\mathcal{E}_{g}(H)=\left\{\begin{array}{c}
(1,1,1),(1,1,2),(1,2,3),(1,2,\{1,2\}),(\{1,2\}, 3, H), \\
(\{1,2\},\{1,2\}, H),(\{1,2\},\{1,2\},\{1,2\}),(H, H, H)
\end{array}\right\} .
$$

We recall the following definition (see [1]).

Definition 3.1 Let $(H, *)$ be a hypergroupoid. An element $e \in H$ is said to be an identity if:

$$
\forall x \in H, \quad x * e \ni x \in e * x
$$

$e$ is said to be a scalar identity if:

$$
\forall x \in H, \quad x * e=\{x\}=e * x .
$$

Now we can prove the following theorem.

Theorem 3.7 Let $(H, *)$ be a commutative semi-hypergroup. Then $(\mathcal{E}(H),+)$ is a commutative semigroup. Moreover, if $(H, *)$ has a scalar identity, then $(\mathcal{E}(H),+)$ has an identity.

Proof. By the previous proposition $(\mathcal{E}(H),+)$ is a groupoid. Let $f, g, k \in$ $\mathcal{E}(H), x \in H$; we have:

$$
(f+(g+k))(x)=f(x) *(g+k)(x)=f(x) *(g(x) * k(x)),
$$

and:

$$
((f+g)+k)(x)=((f+g)(x)) * k(x)=(f(x) * g(x)) * k(x) ;
$$

since $(H, *)$ is a semi-hypergroup, it follows that:

$$
f(x) *(g(x) * k(x))=(f(x) * g(x)) * k(x),
$$

and so $(\mathcal{E}(H),+)$ is a semigroup. Moreover:

$$
(f+g)(x)=f(x) * g(x)=g(x) * f(x)=(g+f)(x),
$$


and so $(\mathcal{E}(H),+)$ is commutative. Finally let $e$ be the scalar identity of $(H, *)$ and let $0_{H}: H \rightarrow \mathcal{P}^{*}(H)$ defined by setting:

$$
\forall x \in H, \quad 0_{H}(x)=\{e\} ;
$$

we have:

$$
\forall x, y \in H, \quad 0_{H}(x * y)=e=e * e=0_{H}(x) * 0_{H}(y),
$$

and so $0_{H}$ is a (good) multiendomorphism; moreover:

$$
\forall f \in \mathcal{E}(H), \forall x \in H, \quad\left(0_{H}+f\right)(x)=0_{H}(x) * f(x)=e * f(x)=f(x),
$$

and, by the commutativity:

$$
\forall f \in \mathcal{E}(H), \forall x \in H, \quad\left(f+0_{H}\right)(x)=f(x) .
$$

So, $0_{H}$ is the identity of $(\mathcal{E}(H),+) . \diamond$

Now we introduce in $\mathcal{E}(H)$ (and so in $\mathcal{E}_{g}(H)$ ) a relation $\leq$ by setting:

$$
\forall f, g \in \mathcal{E}(H), \quad f \leq g \Leftrightarrow f(x) \subseteq g(x), \forall x \in H
$$

Remark 3.2 It is clear that $\leq$ is a partial order in $\mathcal{E}(H)$, that is $(\mathcal{E}(H), \leq)$ is a partially ordered set.

Now we can show the main theorem.

Theorem 3.8 Let $(H, *)$ be a commutative semi-hypergroup. Then $(\mathcal{E}(H),+, \circ, \leq)$ is a $G$-semiring. Moreover, if $(H, *)$ has a scalar identity, then $(\mathcal{E}(H),+, \circ, \leq)$ is with identities.

Proof. By Theorem 3.7 it follows that $(\mathcal{E}(H),+)$ is a semigroup; moreover, if $(H, *)$ has a scalar identity, then $(\mathcal{E}(H),+)$ has an identity $1_{H}$. Now let $f \leq g$ that is:

$$
\forall x \in H, \quad f(x) \subseteq g(x)
$$

we have:

$$
\forall k \in \mathcal{E}(H), \forall x \in H, \quad f(x) * k(x) \subseteq g(x) * k(x)
$$

that is:

$$
\forall k \in \mathcal{E}(H), \quad f+k \leq g+k ;
$$

by the commutativity of + it follows that:

$$
\forall k \in \mathcal{E}(H), \quad k+f \leq k+g .
$$

This shows that $(\mathcal{E}(H),+, \leq)$ is a partially ordered semigroup. 
By Theorem 3.5 it follows that $(\mathcal{E}(H), \circ)$ is a semigroup with identity $0_{H}$. Now let $f \leq g$ that is:

$$
\forall x \in H, \quad f(x) \subseteq g(x)
$$

we have:

$$
\forall k \in \mathcal{E}(H), \forall x \in H, \quad k(f(x)) \subseteq k(g(x)), \quad f(k(x)) \subseteq g(k(x)) ;
$$

that is:

$$
\forall k \in \mathcal{E}(H), \quad k \circ f \leq k \circ g, \quad f \circ k \leq g \circ k ;
$$

This shows that $(\mathcal{E}(H), \circ, \leq)$ is a partially ordered semigroup.

Clearly $0_{H} \neq 1_{H}$. So, it remains to show S.3.

Let $f, g, k \in \mathcal{E}(H)$; we have:

$$
(f \circ(g+k))(x)=f((g+k)(x))=f(g(x) * k(x)) ;
$$

by Lemma 3.1 it follows that:

$$
f(g(x) * k(x)) \subseteq f(g(x)) * f(k(x)) ;
$$

since:

$$
f(g(x)) * f(k(x))=(f \circ g)(x) *(f \circ k)(x)=((f \circ g)+(f \circ k))(x)
$$

we obtain that:

$$
(f \circ(g+k))(x) \subseteq((f \circ g)+(f \circ k))(x)
$$

that is:

$$
(f \circ(g+k)) \leq((f \circ g)+(f \circ k)) .
$$

Moreover:

$$
\begin{aligned}
((f+g) \circ k)(x) & =(f+g)(k(x))=\bigcup_{z \in k(x)}((f+g)(z))=\bigcup_{z \in k(x)}(f(z) * g(z)) \subseteq \\
& \subseteq \bigcup_{z, t \in k(x)}(f(z) * g(t))=\left(\bigcup_{z \in k(x)} f(z)\right) *\left(\bigcup_{t \in k(x)} g(t)\right)= \\
& =f(k(x)) * g(k(x))=(f \circ k)(x) *(g \circ k)(x)= \\
& =((f \circ k)+(g \circ k))(x)
\end{aligned}
$$

that is:

$$
((f+g) \circ k) \leq((f \circ k)+(g \circ k)) .
$$

So, the theorem is proved. $\diamond$

The $G$-semiring obtained in Theorem 3.8 will be called the $G$-semiring of multiendomorphisms.

The following example shows that there exist $G$-semirings that are not semirings. 
Example 3.3 Let $(H, *)$ be the hypergroupoid defined by the following table:

\begin{tabular}{c|c|c|}
$*$ & 1 & 2 \\
\hline 1 & 1 & 1 \\
\hline 2 & 1 & 1,2 \\
\hline
\end{tabular}

We have:

$$
\mathcal{E}(H)=\{(1,1),(1,2),(1, H),(2,2),(2, H),(H, 2),(H, H)\} .
$$

We consider $f=(2, H), g=(H, 2), k=(1, H), t=(H, H)$. We have:

$$
\begin{aligned}
& (g+k)(1)=g(1) * k(1)=H * 1=1 \\
& (g+k)(2)=g(2) * k(2)=2 * H=H .
\end{aligned}
$$

that is:

$$
g+k=k
$$

and so:

$$
f \circ(g+k)=f \circ k=f .
$$

On the other hand:

$$
(f \circ k)+(g \circ k)=f+t=t .
$$

Since, clearly, $f<t$ we have that:

$$
f \circ(g+k)<(f \circ g)+(f \circ k)
$$

and so $(\mathcal{E}(H),+, \circ, \leq)$ is a $G$-semiring that is not a semiring.

\section{End-closed and End -closed semi-hypergroups}

In general, if $(H, *)$ is a commutative semi-hypergroup, the structure $(\mathcal{E}(H),+, \circ)$ is not a semiring, since in general the distributive law does not holds (see example 3.3). Now we give the following definition.

Definition 4.1 A commutative semi-hypergroup $(H, *)$ is said to be Endclosed if and only if $(\mathcal{E}(H),+, \circ)$ is a semiring.

By Remark 2.2 it follows that:

Proposition 4.1 A commutative semi-hypergroup $(H, *)$ is End-closed if and only if:

$$
\forall f, g, k \in \mathcal{E}(H), \quad\left\{\begin{array}{l}
(f \times g)+(f \times k) \leq f \times(g+k) \\
(f \times k)+(g \times k) \leq(f+g) \times k
\end{array}\right.
$$


Analogously, in general, $\left(\mathcal{E}_{g}(H),+, \circ\right)$ is not a semiring since, in general, $\mathcal{E}_{g}(H)$ is not closed under + (see example 3.2). Now we give the following definition:

Definition 4.2 A commutative semi-hypergroup $(H, *)$ is said to be End E- $^{-}$ closed if and only if $\left(\mathcal{E}_{g}(H),+, \circ\right)$ is a semiring.

By using a similar argument as in Theorem 3.8, and by observing that in this case all the inclusions are, in fact, identities, we can prove that:

Proposition 4.2 A commutative semi-hypergroup $(H, *)$ is End Elosed $_{\text {-clo }}$ and only if $\left(\mathcal{E}_{g}(H),+\right)$ is a groupoid.

Clearly, if $(H, *)$ is a commutative semigroup (i.e. if $*$ is single-valued), in the proof of Theorem 3.8 all the inclusions are, in fact, identities, and so we obtain the following corollary.

Corollary 4.3 Any commutative semigroup is End-closed and End $d_{g}$-closed.

Now we give a class of semi-hypergroups (in fact, hypergroups) that are End-closed and End $_{g}$-closed.

Lemma 4.4 Let $(H, *)$ be a commutative semi-hypergroup. If $\mathcal{E}_{g}(H)=$ $\mathcal{E}(H)$ then $(H, *)$ is End-closed and End -closed, that is $\left(\mathcal{E}_{g}(H),+, \circ\right) \equiv$ $(\mathcal{E}(H),+, \circ)$ is a semiring.

Proof. By Proposition 3.7 we know that $(\mathcal{E}(H),+)$ (and so $\left.\left(\mathcal{E}_{g}(H),+\right)\right)$ is a groupoid. The lemma follows now by Proposition 4.3. $\diamond$

Theorem 4.5 Let $(H, *)$ be defined by:

$$
\forall x, y \in H, \quad x * y=\{x, y\} .
$$

Then $(H, *)$ is End-closed and End Elosed. $^{-}$

Proof. First of all it is clear that $(H, *)$ is a commutative hypergroup. Now it is enough to show that in this case $\mathcal{E}_{g}(H)=\mathcal{E}(H)$. We will show that, in fact, any map $f: H \rightarrow \mathcal{P}^{*}(H)$ is a good multiendomorphism. Let $f: H \rightarrow \mathcal{P}^{*}(H)$; we have:

$$
\forall x, y \in H, \quad f(x * y)=f(\{x, y\})=f(x) \cup f(y)=f(x) * f(y),
$$

that is $f$ is a good multiendomorphism. The theorem follows now from Lemma 4.4. $\diamond$

We recall now that a $k$-Steiner system is a geometric space $(H, \mathcal{L})$, where $H$ is a non-empty set, whose elements are called points, and $\mathcal{L}$ is a family of non-empty subsets of $H$, whose elements are called lines, such that every line has $k$ points and any two distinct points are contained in a unique line. In [7] the following definiton was introduced: 
Definition 4.3 A hypergroupoid $(H, *)$ is said to be a $k$-Steiner hypergroupoid $(k \geq 2)$ (or simply Steiner hypergroupoid), if and only if the following conditions are satisfied:

1) $\forall x, y \in H, \quad\{x, y\} \subseteq x * y$;

2) $\forall x, y \in H, \quad|x * y|=\left\{\begin{array}{lll}1 & \text { if } & x=y \\ k & \text { if } & x \neq y\end{array}\right.$;

3) the associativity holds for any triple of points not all distinct.

We can prove that if $(H, \mathcal{L})$ is a $k$-Steiner system then it is possible to define uniquely a $k$-Steiner hypergroupoid $(H, *)$ (see [7], [3], [4] for more details); this $k$-Steiner hypergroupoid is said to be associated to $(H, \mathcal{L})$; in particular, if $k=2$, then the 2-Steiner systems are complete graphs and the 2-Steiner hypergroupoids verify the condition:

$$
\forall x, y \in H, \quad x * y=\{x, y\}
$$

Therefore we can rewrite the previous theorem as follows:

Theorem 4.6 Any Steiner hypergroupoid associated to a complete graph is

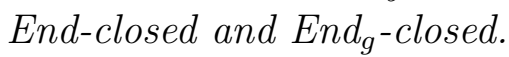

\section{References}

[1] Corsini P., Prolegomena of hypergroup theory, Aviani Editore, Udine, 1993.

[2] Dacić R., Milić S., On multihomomorphisms, Publ. Inst. Math. Beograd, t. 12 (26), 1971, pp. 31-35.

[3] Gentile G., Algebraic and geometric automorphisms of hypergroupoids, Le Matematiche, Catania, Vol. LIII (1998), Fasc. I, pp. 85-105.

[4] Gentile, G., A hypergroupal characterization of projective planes, Discrete Mathematics, 200, 1-3, (2000), pp. 89-105.

[5] Golan J.S., Theory of semiring with applications in mathematics and theoretical computer science, Longman Scientific \& Technical, 1990.

[6] Fuchs L., Partially ordered algebraic systems, International Series of Monographs in Pure and Applied Mathematics, 1963.

[7] Tallini G., Ipergruppoidi di Steiner e Geometrie Combinatorie, Atti del Convegno su Sistemi Binari e Applicazioni, Taormina, (1978).

Received: July 1, 2001 